\title{
Dynamic Analysis Related with Safety for Three Wheeler
}

\author{
W.K.R. Peiris, K.Y.H.D. Shantha and S.A.P.S. Silva
}

\begin{abstract}
Three Wheeler is a very popular vehicle in passengers transporting as well as goods delivering in Sri Lanka. The statistics show that the accidents relate to three-wheeler are becoming significantly exceeding its proportionality in transportation sector. The simple kinematics of threewheeler has reduced its cost but also has increased its instability. The triangular wheel base has increased the instability of three wheeler above two wheels and four wheelers. The intention of this paper is to identify and classify the main modes of accidents related to three wheeler and to develop a dynamic simulation model to mimic the behaviour of a three wheeler under various driving torques, turning radius, topology, location of centre of gravity. Then from this simulation it is interfered basic safe driving conditions.
\end{abstract}

Keywords: Toppling, turning radius, instantaneous centre of rotation

\section{Introduction}

Most people in urban areas as well as rural areas uses three wheelers for their day to day travelling. Low cost and easy maintenance are the main reasons for this. But statistics shows[1][2] that the accidents related to three wheelers are also at an alarming level. Three wheel drivers are also from the general society therefore it is not possible to rule them as a special non disciplined group in driving and therefore these accidents cannot be debited to the misbehaviour of the drivers. Therefore the intension of this study was to analyse the physical modes of accidents in three wheelers and to develop a simulation method to analyse them so as to introduce relevant safety measures.

In the literature survey it was revealed that there are standard testing procedures for dynamic testing of three wheelers[3],[4],[5],[2]. But all these specific tests have been developed to examine specific safety conditions such as roll over protection dismiss of controllability etc. under limiting conditions. In this study a dynamic model from basics will be developed to analyze the behavior of a three wheeler in a flat terrain under any kinematics/dynamics conditions and with the facility of including any feature, such as time varying steering inputs, effects of change of camber angle, mass distribution pattern etc. (Considering the Centre of mass as the point concentrating all the masses of the system provides a poor dynamic model)

The main driving parameters such as acceleration, braking and turning should have to be considered in analyzing the behavior of a vehicle. In general the time variation of the above said driving parameters will govern the behavior of the vehicle (time-position relation) Therefore in avoiding an accident (collision) these parameters are very much important.

For avoiding an accident (collision) the above parameters will have to vary within their limits (varied by the driver) in such a way that the obstacle would be avoided without toppling of the vehicle.

Safe driving conditions for any vehicle could be identified as conditions under which the driver has the control over the center of rotation and acceleration/deceleration (within power/brake limits of the vehicle) of the vehicle. When over/under steering, slipping and toppling are taking place driver loosen this control, hence safety driving conditions diminishes.

In a normal vehicle with pneumatic tires, parameters such as speed of the vehicle, time variations of the steering angle of the front wheel, centrifugal forces, cornering forces on tires, braking/ accelerating forces on tires, tire reactions on road, suspension characteristics, mass distribution of the vehicle, wheel base of the vehicle and slip angle characteristics of tires will play an vital role in such situation.

When analyzing the kinematic/dynamic aspects of accidents related to three wheelers, it was observed that almost all the accidents could be characterized into four major groups (four modes of accidents)

Eng. W.K.R.Peris, B.Sc.Eng, Mphill, CEng, Research Fellow, MIE(Sri Lanka), NERD Centre.

Eng. K.Y.H.D. Shantha, B.Sc.Eng, MSc, AMIE(Sri Lanka), Director (Technical), NERD Centre

Eng. S.A.P.S. Silva, B.Sc.Eng (First Class Hons), AMIE(Sri

Lanka), ResearchEngineer, NERD Centre. 


\section{Collision with the obstacle without toppling or immediately after toppling}

High speed travelling of three-wheelers, causes the most possible common type of accidents in main roads. This is mainly due to exceeding control limits of the vehicle. There may be two main ways of causing accidents due to above reason. The over steering characteristics of three wheeler, (i.e. at high speeds even for a lower steering angle input the actual turning radii will be less than the radius determined by steering angle and slide slip free condition. (i.e. The turning radius determined by the geometry.)

The other main reason is under steering condition which may take place when the three-wheeler is over loaded in rear end. In this case the driver may not be able to drive the vehicle so as to bypass the obstacle.
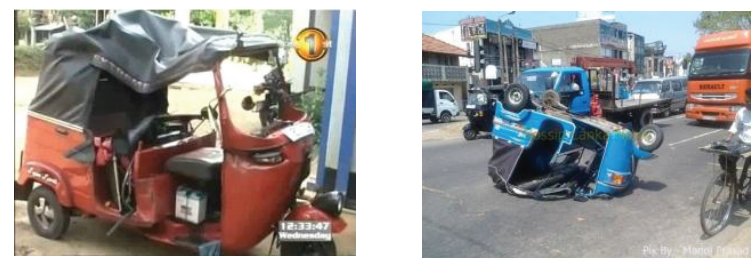

Figure 1 - Three wheeler accidents due to toppling
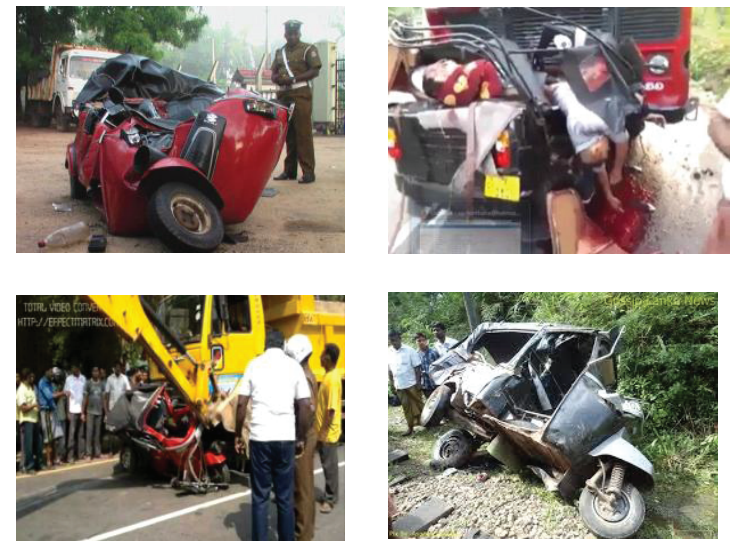

Figure 2 - Three wheeler accidents due to hitting while tending for toppling-Toppling condition is evident from the damages to hood

From the photos taken at accident places, it is evident that in most cases the cause of accident is toppling. In most of the other cases the damage may be to either side or hood of the three-wheeler. The main cause is hitting the obstacle while tending to topple.

\section{Just Toppling}

This mode is very common in places where drivers tend to turn the vehicle abruptly for quick-changing of the path of motion. Braking of the lock provided for over steering control, is a major cause for this type of toppling also and almost slip free turning condition would support these situations.

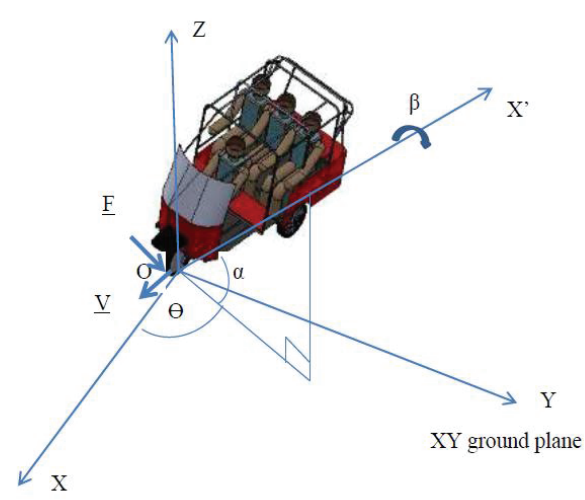

Figure 3 - schematic diagram for analysis of Kinematic of a typical toppling due to sudden turning. $(\underline{V}=$ Velocity of ground contact point of front wheel immediately after sudden turn.)

In a sudden turn an impact type lateral force $(F)$ will act laterally on front wheel.

\section{Therefore}

Equation I

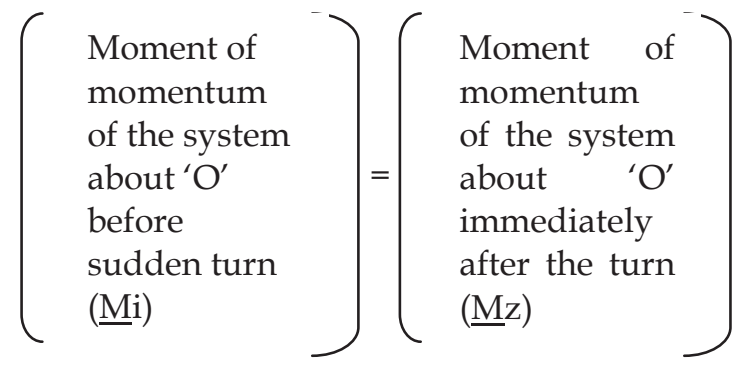

\section{Equation II}

- Linear momentum of the system perpendicular $($ ) to ' $\mathrm{F}$ '

will also be conservative from [I] \&[II] kinematic parameters $(\dot{\theta}, \dot{\alpha}, \dot{\beta}, v)$ could be determined.

\section{Toppling/moving out of the way due to impacts by external bodies such as small vehicles(bikes), dogs, cows.}

There are light impacts and therefore no significant change of the overall shape of the three-wheeler. 


\section{Toppling/moving out of the way due to moving on obstacles with high speeds and relevant turning radii.}

Unlike in normal four wheel type vehicles toppling is the major issue in three wheelers. This is mainly due to its triangular wheel base. Triangular wheel base provides the line of turning which has very high tendency of toppling. In four wheels the tendency of toppling about such line is low. In twowheelers (cycles) although the tendency of toppling is around such line is very high (almost unstable) leaning action taking place in turning prevents toppling. But in three wheelers there is no such leaning action.

When trying to avoid the obstacle by turning, braking/accelerating the toppling is the main constrain. Therefore unlike in the normal vehicle which has very little tendency of toppling because of its rectangular wheel base, it is a critical issue to avoid an obstacle under similar road traffic conditions for a three wheeler when compared with four wheel vehicle.

Therefore it is very much important to analyze the dynamic behavior of three wheelers and to study the relation among said parameters in driving and to introduce suitable relation among those parameters for a safe drive. Also with the study (analysis) it will be possible to develop suitable relationship among dimensional parameters and parameters such as braking, handle to wheel turning characteristics of steering, camber angle in turning of front wheel, suspension characteristics and slide slip characteristics.

\section{Development of a model to analyse the dynamic system related to the three-wheeler}

Figure (4) shows a schematic diagram of a three-wheeler moving with constant angle of steer $(\theta)$ with forces and accelerations which are parallel to the plane in which the vehicle is moving. This plane is inclined to horizontal by " $\gamma$ " and according to figure (4) the instantaneous travelling direction is given.

When moments about the axis through " $\mathrm{O}$ " perpendicular to the travelling plane is considered. One could obtain equation (3) (with reversed inertial forces). Similarly when the forces(with reversed inertial forces) are resolved in $\mathrm{AD}$ direction one could obtain equation (4). Then with the relations given as (5) ( $\mathrm{k}=$ coefficient of side slip $\approx 0.4$ ) for $F_{f}$ a particular initial configuration given by " $\phi$ " and with initial.

$$
\begin{aligned}
& \theta \quad=\text { steered angle } \\
& \text { C.M = Centre of mass of three-wheeler \& } \\
& \text { passengers } \\
& O=\text { Centre of rotation } \\
& V_{G} \quad=\text { Velocity of Centre of mass } \\
& f_{x}=\text { Inertial periferral acceleration of } \\
& \text { centre of mass } \\
& \alpha \quad=\text { Side slip angle } \\
& \mathrm{f}_{\mathrm{y}} \quad=\text { Centripital acceleratin of centre of mass } \\
& T=\text { Traction force on rear wheels } \\
& F_{r} \quad=\text { Side force on rear wheels } \\
& F_{f}=\text { Side force on front wheels } \\
& M=\text { Total mass }
\end{aligned}
$$

The model is based on the following assumptions

i. Effect of suspension is rigid.

ii. Initial effects are concentrated to the center of mass of the system.

iii. Side slip coefficient for front wheel is constant.

iv. Side slip coefficient for rear wheels are zero due to high reaction on rear wheels compared to front wheels.

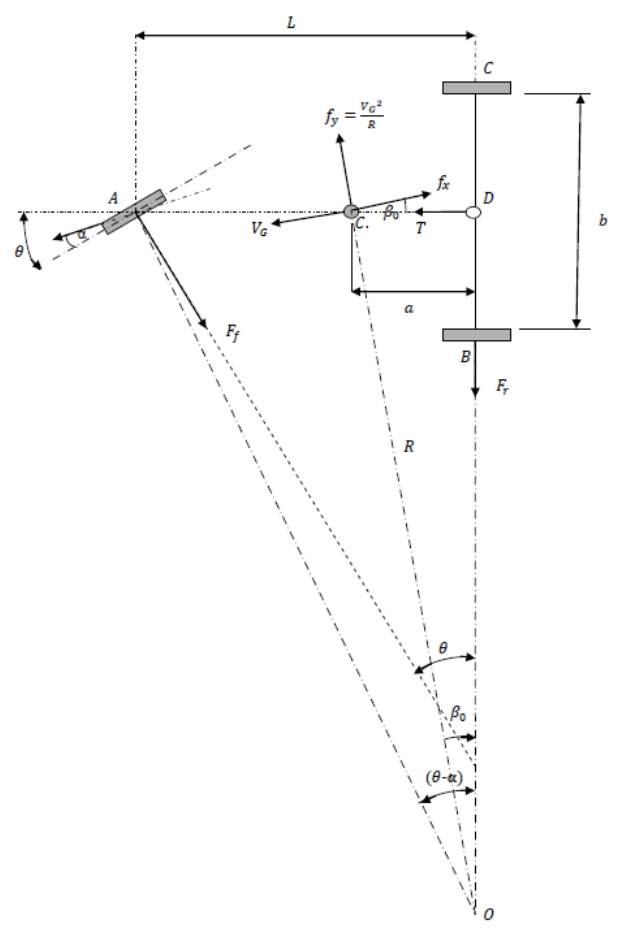

Figure 4 - Schematic diagram of a threewheeler moving with constant angle of steer $(\theta)$ with forces and accelerations 
$M f_{x} \cos \beta_{0}\left(\frac{L}{\tan \theta}\right)+a M f_{x} \sin \beta_{0}+a M f_{y} \cos \beta_{0}-$

$M f_{y} \sin \beta_{0}\left(\frac{L}{\tan \theta}\right)-T\left(\frac{L}{\tan \theta}\right)+$

$M g \sin \gamma \cos \varnothing\left(\frac{L}{\tan \theta}\right)-a M g \sin \gamma \sin \emptyset=0$

$F_{y} \sin \theta+M f_{x} \cos \beta_{0}-\frac{M V_{G}^{2}}{a} \sin ^{2} \beta-T-$

$M g \sin \gamma \cos \emptyset=0$

Where;

$\tan \beta_{0}=\frac{a}{L} \tan \left[\theta-k F_{f}\right]$

$f_{y}=\frac{V_{G}^{2}}{R}, \quad R=\frac{a}{\sin \beta_{0}}$

Solve (1), (2), \& (3); Find $f_{x}, F_{f}$ then

$\left(V_{G}\right)_{n+1}=\left(V_{G}\right)_{n}+\left(f_{x}\right)_{n} \delta t$

\subsection{Determination of forces on wheels}

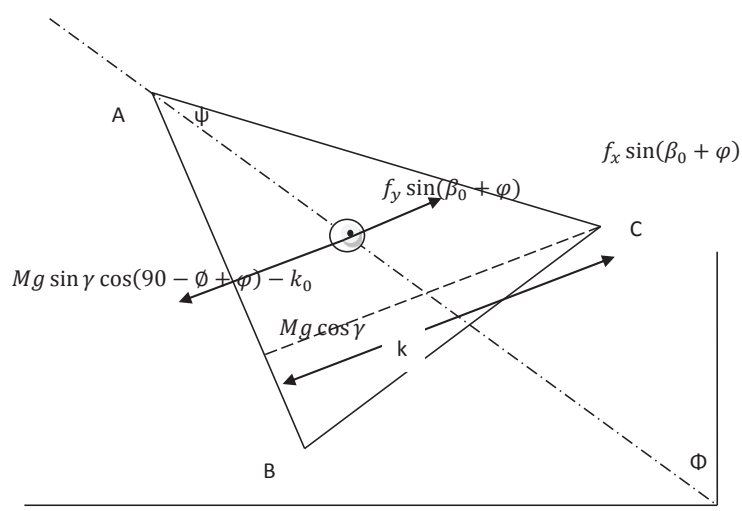

$$
\begin{aligned}
& {[M g \sin \gamma \cos (90-\emptyset+\varphi)} \\
& \left.-M\left(f_{x}+f_{y}\right) \sin \left(\beta_{0}+\varphi\right)\right] H g \\
& -k_{0} M g \cos \gamma+R_{C} k=0
\end{aligned}
$$

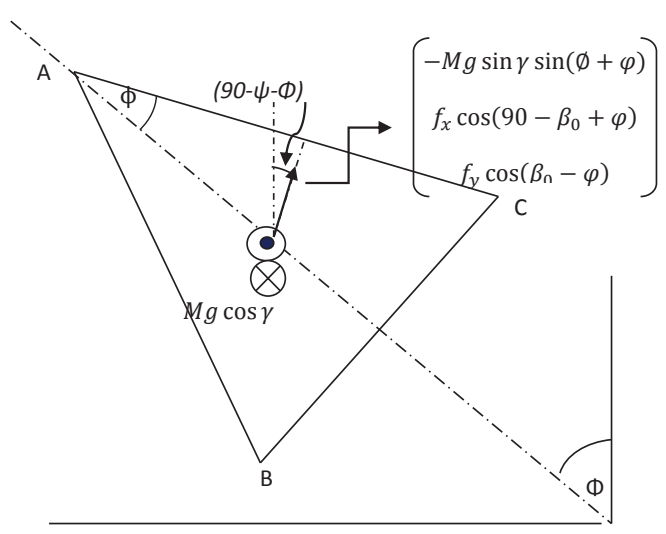

$k R_{B}-k_{0} M g \cos \gamma+H g[(-M g \sin \gamma) \sin (\varnothing+$ $\left.\varphi)+M f_{x} \cos \left(90-\beta_{0}+\varphi\right)+M f_{y} \cos \left(\beta_{0}-\varphi\right)\right]=$ 0

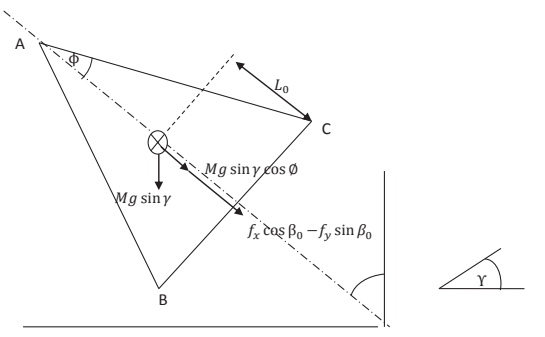

Figure 5- Schematic diagrams of forces

$L R_{A}+H_{g} M g \sin \gamma \cos \emptyset+H_{g} M f_{x} \cos \beta_{0}-$

$H_{g} M f_{y} \sin \beta_{0}-L_{0} M g \cos \gamma=0$

Also by frame resolution

$F_{y} \cos \theta+F_{r}=M f_{y} \cos \beta_{0}+M f_{x} \sin \beta_{0}$

Now

$F_{f} \& F_{r}$ could be determined.

Then side slipping could be inferred.

Now for any initial condition

$\mathrm{R}, \mathrm{z} \quad\left(V_{R}\right)_{0} \& T(t), \quad \gamma=0, \varnothing=0$ (flat land)

$\theta=\theta_{0}$ (not a function of time)

$R_{A}(t), R_{B}(t), R_{C}(t) \& F_{f}(t), F_{r}(t)$ could be determined by aniterative procedure.

also $V_{R}(t)$.

Rolling resistance could be included in " $T$ "

Values of $T_{0}, V_{G_{0}}$. It is possible to determined the values for $f_{x} \& F_{f}$ now with this $f_{x}$,

$V_{G_{1}}=V_{G_{0}}+\delta t f_{x}$

Where

$V_{G_{1}}=$ velocity of centre of mass after $\delta t$ time.

Now with this $V_{G_{1}}$ \& relevant $\mathrm{T}$ values at $\delta \mathrm{t}$, and relevant $\Phi$ values it is possible to determine the $f_{x}$ at $\delta t$.Then with this iterative procedure " $V_{G}$ at any time could be determined, also $F_{y}$ could be determined.

Now with the equations ( 4$),(5),(6) \&(7)$. Reactions on wheels $R_{A}, R_{B}, R_{C}$ could be determined. Toppling could be inferred by $R_{A}, R_{B} \& R_{C}$ which slipping could be inferred by $F_{f} \& F_{r}$ values.

Therefore this model provides a method to infer the reactions\& side forces on wheels at any time after a particular initial condition (velocity) with constant steered angle and time varying traction/brake. Also the kinematics of the model could be easily developed for incooperate time varying steering inputs. Figure $7,8,9$ shows the variation of reactions on front and rear wheels for a particular set of initial conditions and geometric parameters.

From the above model reaction on each wheel could be inferred at any time for given set of initial conditions. Here rolling resistance and brake forces (significant only for rear wheels also could be included in traction term ' $\mathrm{T}$ '. Now $\mathrm{F}_{\mathrm{f}} \& \mathrm{~F}_{\mathrm{r}}$ (side forces on front and rear wheels respectively) could be determined by simple force analysis for the system. If $R_{A}, R_{B}, R_{C}<0$ toppling conditions will occur. If $F_{f}, F_{r}$ exceeds 
relevant frictional forces slipping will occur. Therefore with this model the safe behaviour of three wheelers could be inferred with these inferred information a dynamic testing procedure for three wheelers could be introduced.

\section{Flow chart for analysis}

The developed flow chart to simulate the system (with visual basic), as stated in section 5 this solution -iterative algorithm is for a fixed steering angle $(\theta)$

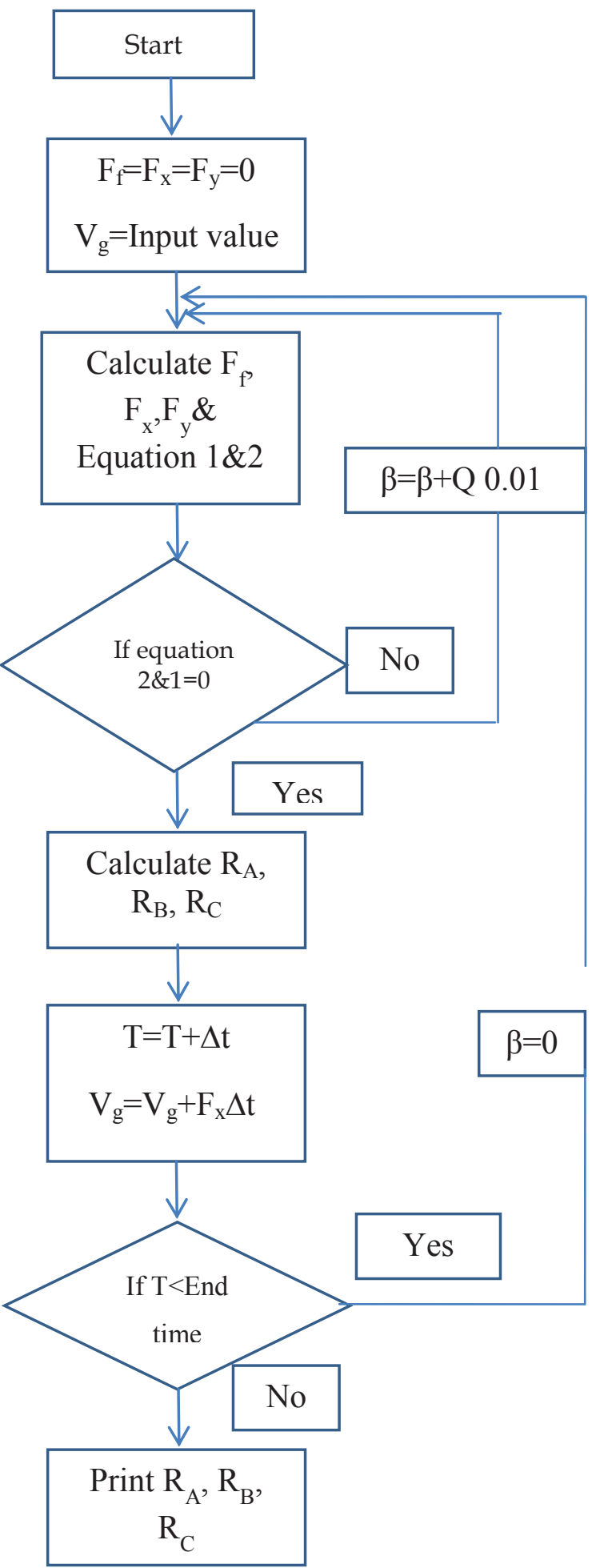

\section{NOTE}

The following are the results obtained for wheel reactions from the model for the below given initial condition. Safety conditions of the vehicle could be inferred by the values as stated in 5.1

Input values for program

$\mathrm{M}=700 \mathrm{~kg}$

$\mathrm{Vg}$ initial $=8.5 \mathrm{~m} / \mathrm{s}$

$\mathrm{L}=1.97 \mathrm{~m}$

$\mathrm{a}=0.39 \mathrm{~m}$

$\gamma=0 \quad \theta=30^{\circ}$

$\phi=0$

Program is in annexes

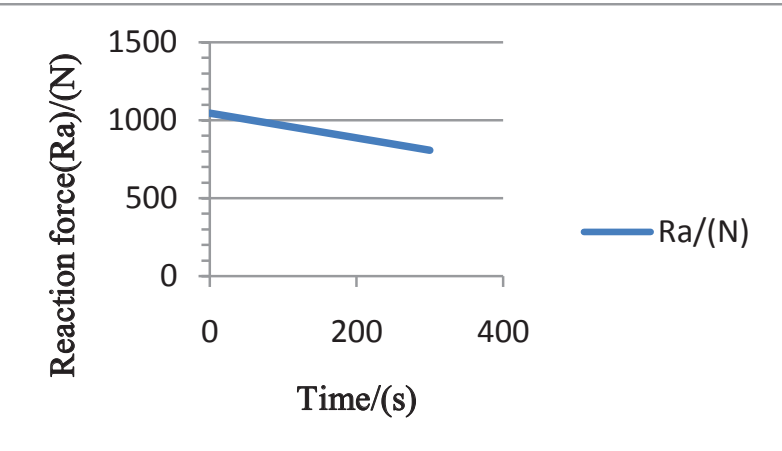

Figure 7 - Front wheel Reaction force $\left(R_{a}\right)$ vs time

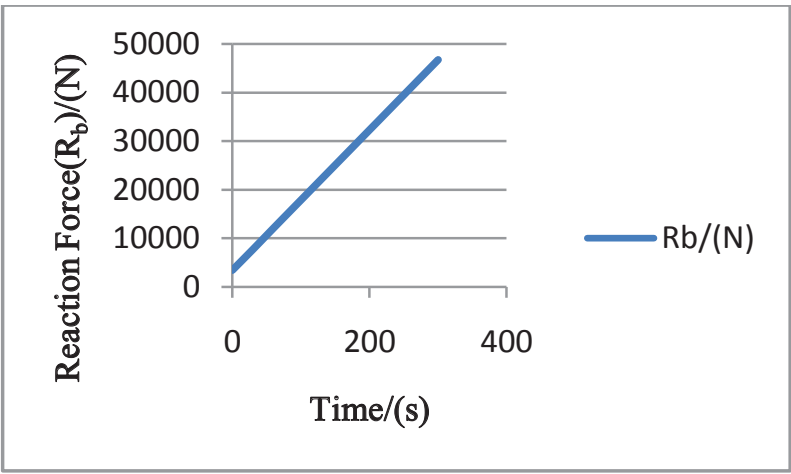

Figure 8 - Rear left side wheel reaction force $\left(R_{b}\right)$ vs time

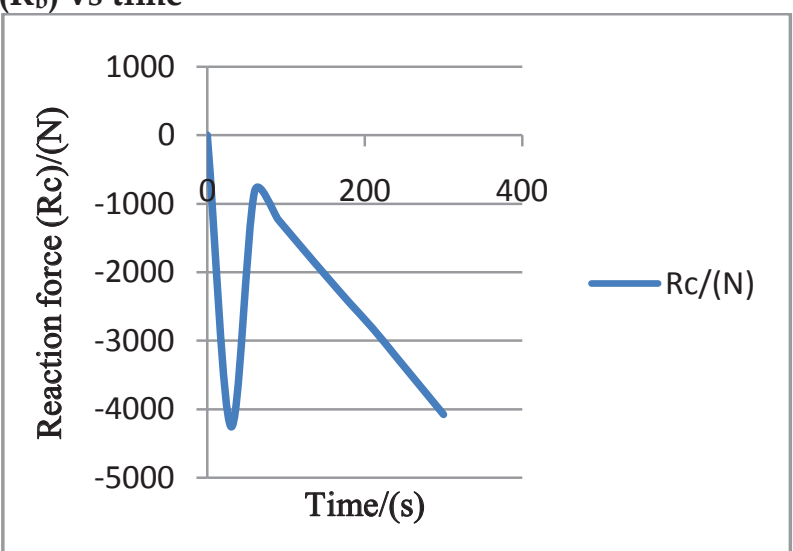

Figure 9 - Rear right side wheel reaction force $\left(\mathbf{R}_{c}\right)$ vs time

Figure 6 - Flow chart for simulation 


\section{Conclusion}

The kinematic analysis of three wheeler accidents shows that in most cases toppling or toppling tendencies have been taken place before the impact(Hitting on the other vehicle/obstacle).In most accidents which were analysed simultaneous turning and braking has resulted in toppling. With braking due to sudden deceleration reaction on rear wheels will be decreased and these wheels will slip side verse giving rise to over steered conditions (i.e. Reducing the actual turning radius) which will tend the vehicles to topple. In some other cases turning with high acceleration will give rise to under steered conditions. With acceleration reaction on front wheel will be reduced and hence the side verse slip on front wheel will be decreased resulting in under steered conditions. In these situations the driver will not be able to bypass the obstacle.

All these conditions could be simulated with the above model. Therefore by studying the model by including real values for relevant parameters in the model few dynamic testing procedures could be introduced.

Although the analysis in section five is for a fixed steering angle it could be approximated for a slow variation of steering angle with the introduction of change of angle for each step. In that case if the reduction in steering angle is slow, when angle become small (low turning radius) speed will also be much low due to rolling resistance (and braking) therefore less possibility of toppling. But if the rate of change of angle is high the turning radius will become low at high speed and tendency for toppling will be high.

Therefore it could be concluded that for a three wheeler a steering wheel type turning will decrease the tendency of toppling. Therefore it is recommended to introduce a mechanism with slow steering response instead of the handle bar which gives a one to one steering response.

The reliability of the results of the model will depend on the validity of the values of friction/rolling resistance coefficient, the fitness of the mass discretising grid (In the sample calculation the total mass is concentrated at the centre of mass of the system) and the fines of the time $(\delta \mathrm{t})$ discretization. The convergence of the model in the time domain is satisfactory up to $\delta \mathrm{t}=0.01 \mathrm{~s}$.

The scope of this paper was only to develop a kinematic/dynamic model with basic mechanics for three wheelers under particular conditions (sec 5.1) and to form an iterative procedure to solve the same. A sample calculation is introduced for wheel reactions. Although negative reaction for wheels are practically meaningless, the model will not restrict such results and whenever reactions become negative it will simply mean that the toppling is taking place. And therefore the condition at which the toppling will take place (i.e. condition at which one of the reaction just becomes zero) could be inferred from the model. Similarly side forces (on wheels) also could be determined from the model and slipping could be inferred.

Authors hardly see the requirement of "validating" the model through practical testing facilities, because it is almost based on basic Newtonian mechanics (assuming rolling/friction coefficient are reliable) and does not involve with any vague empirical type equations as such. Therefore if convergence is satisfied the model could be straight away used to infer the behaviour of three wheels and thereby safety driving conditions could be inferred.

\section{Acknowledgement}

We acknowledge Vijaya Publications which publishes Sunday Times and Lankadeepa for providing us valuable articles in related to the topic of safety aspects of three wheelers and their effects on socio economic conditions of Sri Lankan people. We also acknowledge to Department of Motor Traffic for providing us valuable information on three wheelers and details of manufacturers with specifications of different brands.

Finally we should be acknowledge and grateful to National Engineering Research and Development Centre of Sri Lanka where we are employed for allowing us to continue this study and allocating the resourced environment.

\section{References}

[1] "http://wenku.baidu.com/ view/1bcdff8884868762caaed5b0.html," [Online].

[2] Staee, P. P. J., "Designing stable three wheel vehicles," no. university of Minnesota.

[3] Chawla, A., Mukheerjee, S., and Nayak, A., "Investigating the rollover propensity of a 15 seat mini bus," Vehical Safety, vol. 02, 2007.

[4] Ellis, J. R., Vehicle dynamics, London: Business Books, 1969 November.

[5] Mukheerjee, S. and Mohan, D.:T.R.Gawale, "Three Wheeler scooter taxi:A safety Analysis," Sadhana, vol. 32, no. India, pp. 459-478, August 2007. 
[6] Mukheerjee, S., Mohan, D., and Gawale, T. R., "Rollover crash analysis of the RTV using Madymo," no. Indian Institute of Technology,India, 2005.

\section{Annexes \\ Program for simulation}

PPrivate Sub CommandButton1_Click()

Dim M As Single

Dim T As Single

Dim K As Single

Dim a As Single

Dim K0 As Single

Dim b As Integer

Dim FuiAs Single

Dim RFuiAs Single

Dim shai As Single

Dim RshaiAs Single

Dim Gama As Single

Dim Theta As Single

Dim rtheta As Single

Dim Traction As Single

Dim L As Single

Dim L0 As Single

Dim Vg As Single

Dim Hg As Single

Dim Ra As Single

Dim RbAs Single

Dim Rc As Single

Dim FinalTAs Single

Dim Dt As Single

Dim beta As Double

Dim CbetaAs Double

Dim Fx As Single

Dim Fy As Single

Dim FfAs Double

Dim eqn4 As Single

$\mathrm{M}=\mathrm{Val}$ (TextBox1.Text)

$\mathrm{K}=\mathrm{Val}($ TextBox10.Text)

$\mathrm{Hg}=\mathrm{Val}($ TextBox13.Text)

$\mathrm{K} 0=\mathrm{Val}($ TextBox14.Text $)$

$\mathrm{L} 0=\mathrm{Val}($ TextBox15.Text)

Traction $=\operatorname{Val}($ TextBox16.Text $)$

$\mathrm{Vg}=\mathrm{Val}($ TextBox17.Text)

Fui $=$ Val (TextBox3.Text $)$

RFui $=$ Fui $* 0.01744444$

Gama $=$ Val $($ TextBox4.Text $)$

rgama $=$ Gama * 0.01744444

Theta $=\operatorname{Val}($ TextBox5.Text $)$

rtheta $=$ Theta $* 0.01744444$

shai $=\operatorname{Val}($ TextBox12.Text $)$

Rshai $=$ Theta $* 0.01744444$

$\mathrm{L}=\operatorname{Val}($ TextBox6.Text)

$\mathrm{a}=\operatorname{Val}($ TextBox11.Text)

$\mathrm{Dt}=\mathrm{Val}($ TextBox9.Text)

FinalT $=60 * \operatorname{Val}($ TextBox8.Text $)+1$

$\mathrm{T}=0$

beta $=0$ $b=0$
$R a=0$
$R b=0$
$R c=0$
Do

Do

$\mathrm{Ff}=(-\operatorname{Atn}((\mathrm{L} / \mathrm{a})$ * Tan $($ beta $))+$ rtheta $) /(\mathrm{K})$

$\mathrm{Fy}=\mathrm{Vg} * \mathrm{Vg} * \operatorname{Sin}($ beta $) /(\mathrm{a})$

$\mathrm{Fx}=((\mathrm{Ff} * \operatorname{Sin}($ rtheta $))-(\mathrm{T})-(\mathrm{M} * 9.81$ *

$\operatorname{Sin}($ rgama $){ }^{*} \operatorname{Cos}($ RFui $\left.)\right)-\left((\mathrm{M} / \mathrm{a}){ }^{*} \mathrm{Vg} * \mathrm{Vg}\right.$ *

$\operatorname{Sin}($ beta $) * \operatorname{Sin}($ beta $))) /(-1 * M$ * $\operatorname{Cos}($ beta $))$

eqn $4=(M * F x * \operatorname{Cos}($ beta $) * \mathrm{~L})+($ Tan $($ rtheta $)$ *

$\mathrm{a}{ }^{*} \mathrm{M} * \mathrm{Fx} * \operatorname{Sin}($ beta $\left.)\right)+\left(\operatorname{Tan}(\right.$ rtheta $){ }^{*} \mathrm{a}{ }^{*} \mathrm{M} * \mathrm{Fy}$

* $\operatorname{Cos}($ beta $))-(\mathrm{M} * \mathrm{Fy}$ * $\operatorname{Sin}($ beta $) * \mathrm{~L})-($ Traction

* $\mathrm{L})+(\mathrm{M}$ * 9.81 * $\operatorname{Sin}($ rgama $)$ * $\operatorname{Cos}($ RFui $)$ * $\mathrm{L})-$

(Tan $($ rtheta $) * a * M * 9.81$ * $\operatorname{Sin}($ rgama $)$ *

Sin(RFui))

eqn $4=(\text { eqn } 4 \wedge 2)^{\wedge} 0.5$

beta $=$ beta +0.01

eqn $4=($ eqn $4 \wedge 2) \wedge 0.5$

Loop While eqn $4>0.01$

$\mathrm{b}=\mathrm{b}+1$

Cbeta $=$ beta -0.01

$R_{C}=((M * 9.81 * \operatorname{Sin}($ rgama $) * \operatorname{Cos}(1.57-$ RFui +

Rshai $))-(\mathrm{M}$ * $(\mathrm{Fx}+\mathrm{Fy})$ * Sin (beta + Rshai $))$ * $(-1$

* $\mathrm{Hg} / \mathrm{K})$

$\mathrm{Rb}=((\mathrm{K} 0$ * $\mathrm{M}$ * 9.81 * $\operatorname{Cos}($ rgama $))-((-1$ * $\mathrm{M}$ *

9.81 * Sin $($ rgama $) * \operatorname{Sin}($ RFui + Rshai $))+(M * F x$

${ }^{*} \operatorname{Cos}(1.57$ - beta + Rshai $\left.)\right)+(\mathrm{M}$ * Fy * Cos(beta -

Rshai $)) *(\mathrm{Hg})) /(\mathrm{K})$

$\mathrm{Ra}=((\mathrm{L} 0$ * $\mathrm{M}$ * 9.81 * $\mathrm{Cos}($ rgama $))+(\mathrm{Hg}$ * $\mathrm{M}$ *

$\mathrm{Fy} * \operatorname{Sin}($ beta $))-(\mathrm{Hg} * \mathrm{M} * 9.81$ * $\operatorname{Sin}($ rgama $)$ *

$\mathrm{Cos}($ RFui $))-\left(\mathrm{Hg} * \mathrm{M} * \mathrm{Fx}{ }^{*} \operatorname{Cos}(\right.$ beta $\left.\left.)\right)\right) /(\mathrm{L})$

Cells $(b+7,1)=b-1$

Cells $(b+7,2)=\mathrm{T}$

Cells $(b+7,3)=$ beta -0.01

Cells $(b+7,4)=V g$

Cells $(b+7,5)=$ Fx

Cells $(b+7,6)=$ Fy

Cells $(b+7,7)=$ Ff

Cells $(b+7,8)=$ eqn4

Cells $(b+7,9)=R a$

Cells $(b+7,10)=R b$

Cells $(b+7,11)=$ Rc

$\mathrm{T}=\mathrm{T}+(\mathrm{Dt})$

$\mathrm{Vg}=\mathrm{Vg}+(\mathrm{Fx} * \mathrm{Dt})$

beta $=0$

$\mathrm{Ff}=0$

$\mathrm{Fx}=0$

$\mathrm{Fy}=0$

$\mathrm{Ra}=0$

$\mathrm{Rb}=0$

$\mathrm{Rc}=0$

eqn $4=500$

Loop While $\mathrm{T}<$ FinalT

End Sub 